\title{
RESEARCH
}

Open Access

\section{Radiation-induced toxicity in rectal epithelial stem cell contributes to acute radiation injury in rectum}

Felipe Rodriguez Tirado ${ }^{1}$, Payel Bhanja ${ }^{1}$, Eduardo Castro-Nallar ${ }^{2}$, Ximena Diaz Olea ${ }^{1}$, Catalina Salamanca ${ }^{1}$ and Subhrajit Saha ${ }^{1,3^{*}}$ D

\begin{abstract}
Background: Radiation-induced rectal epithelial damage is a very common side effect of pelvic radiotherapy and often compromise the life quality and treatment outcome in patients with pelvic malignancies. Unlike small bowel and colon, effect of radiation in rectal stem cells has not been explored extensively. Here we demonstrate that Lgr5-positive rectal stem cells are radiosensitive and organoid-based transplantation of rectal stem cells mitigates radiation damage in rectum.
\end{abstract}

Methods: C57Bl6 male mice (JAX) at $24 \mathrm{~h}$ were exposed to pelvic irradiation (PIR) to determine the radiation effect in pelvic epithelium. Effect of PIR on Lgr5-positive rectal stem cells (RSCs) was determined in Lgr5-EGFP-Cre-ERT2 mice exposed to PIR. Effect of PIR or clinically relevant fractionated PIR on regenerative response of Lgr5-positive RSCs was examined by lineage tracing assay using Lgr5-eGFP-IRES-CreERT2; Rosa26-CAG-tdTomato mice with tamoxifen administration to activate Cre recombinase and thereby marking the ISC and their respective progeny. Ex vivo three-dimensional organoid cultures were developed from Lgr5-EGFP-Cre-ERT2 mice. Organoid growth was determined by quantifying the budding crypt/total crypt ratio. Organoids from Lgr5-EGFP-ires-CreERT2-TdT mice were transplanted in C57BI6 male mice exposed to PIR. Engraftment and repopulation of Lgr5-positive RSCs were determined after tamoxifen administration to activate Cre recombinase in recipient mice. Statistical analysis was performed using Log-rank (Mantel-Cox) test and paired two-tail $t$ test.

Result: Exposure to pelvic irradiation significantly damaged rectal epithelium with the loss of Lgr5+ve rectal stem cells. Radiosensitivity of rectal epithelium was also observed with exposure to clinically relevant fractionated pelvic irradiation. Regenerative capacity of Lgr5+ve rectal stem cells was compromised in response to fractionated pelvic irradiation. Ex vivo organoid study demonstrated that Lgr5+ve rectal stem cells are sensitive to both single and fractionated radiation. Organoid-based transplantation of Lgr5+ve rectal stem cells promotes repair and regeneration of rectal epithelium.

Conclusion: Lgr5-positive rectal stem cells are radiosensitive and contribute to radiation-induced rectal epithelial toxicity. Transplantation of Lgr5-positive rectal stem cells mitigates radiation-induced rectal injury and promotes repair and regeneration process in rectum.

Keywords: Rectal stem cell, Pelvic irradiation, Crypt, Mice

\footnotetext{
* Correspondence: ssaha@kumc.edu

'Department of Radiation Oncology, University of Kansas Medical Center, 3901 Rainbow Boulevard, Kansas City, KS 66160, USA

${ }^{3}$ Department of Cancer Biology, University of Kansas Medical Center, 3901

Rainbow Boulevard, Kansas City, KS 66160, USA

Full list of author information is available at the end of the article
}

C C The Author(s). 2021 Open Access This article is licensed under a Creative Commons Attribution 4.0 International License, which permits use, sharing, adaptation, distribution and reproduction in any medium or format, as long as you give appropriate credit to the original author(s) and the source, provide a link to the Creative Commons licence, and indicate if changes were made. The images or other third party material in this article are included in the article's Creative Commons licence, unless indicated otherwise in a credit line to the material. If material is not included in the article's Creative Commons licence and your intended use is not permitted by statutory regulation or exceeds the permitted use, you will need to obtain permission directly from the copyright holder. To view a copy of this licence, visit http://creativecommons.org/licenses/by/4.0/ The Creative Commons Public Domain Dedication waiver (http://creativecommons.org/publicdomain/zero/1.0/) applies to the data made available in this article, unless otherwise stated in a credit line to the data. 


\section{Introduction}

Rectal injury is a major limiting factor for definitive chemo-radiation therapy of pelvic malignancies, such as prostate cancer, bladder cancer, or ovarian cancer, collectively $20-30 \%$ of all malignancies [1, 2]. Thus, effective doses of radiation and/or chemotherapy often cannot be administered, resulting in poor survival and early metastatic spread. Even with stereotactic radiosurgery targeting the tumor, the dose may be limited by an increase in acute rectal epithelial loss leading to acute bleeding followed by chronic proctitis [3]. Radiation proctitis is a common and debilitating consequence of radiation therapy-induced damage to the rectal tissue characterized by acute mucosal loss, inflammation, followed by progressive tissue scarring leading to organ fibrosis. Currently, there are no FDA-approved treatments that can be used to protect against radiation-induced damage to the rectal epithelium. Previous research on radiation-induced toxicity in small bowel demonstrated that radiation-induced loss of intestinal stem cell is the major cause of mucosal damage [4-6]. Unlike radiationinduced small bowel toxicity majority of the reports on radiation-induced rectal injury described radiation-induced proctitis/fibrosis [7, 8]. However, external beam pelvic radiotherapy very frequently causes acute injury such as rectal mucosal damage along with rectal hemorrhage/ bleeding. To date, not much has been known about radiation-induced toxicity in rectal epithelial stem cells.

Adult epithelial stem cells in small bowel are primarily located in crypt base and function as structural building block for repair and regeneration [4, 9]. Crypts present within the mouse small intestine have two types of stem cells. Bmi1-positive intestinal stem cells (ISCs) that are long-lived, label-retaining stem cells present at the +4 position of the crypt base. These Bmi1+ve ISCs interconvert with more rapidly proliferating LRG5+ve stem cells known as CBCs that express markers including Lgr5, Olfm4, Lrig1, and Ascl2 [10-15]. In both in vivo mice model and ex vivo organoid model, it has been demonstrated that sensitivity of these stem cells is the determining factor for mucosal epithelial response to radiation exposure $[4,6]$. In small intestine, it has been reported that label-retaining stem cells present at the +4 position are more radio-resistant than $\mathrm{CBCs}$ and function as a reserve stem cell pool.

In the rectum, the presence of similar stem cell system has not been reported. Moreover, the involvement of these stem cells in regenerative response of rectal epithelium against radiation injury has not been studied well. An extensive literature shows that organoids derived from intestines recapitulate structural and functional characteristics of the tissue of origin. Organoids maintain basic crypt physiology [9] depending on stem cell survival and self-renewal. In the present study, we have demonstrated that radiation-induced loss of Lgr5+ve rectal stem cell is the major cause of rectal epithelial damage. In mice model as well as in ex vivo organoid model, we have demonstrated that exposure to clinically relevant fractionated dose significantly reduces Lgr5+ve rectal stem cell along with epithelial regeneration. Rectal transplantation of rectal organoid in mice exposed to pelvic irradiation demonstrated engraftment of repopulating rectal stem cells and thereby repair and regeneration of rectal epithelium.

\section{Method \\ Animals}

Six- to 10-week-old male C57BL6/J mice, Lgr5-eGFPIRES-CreERT2 mice, B6.Cg-Gt (ROSA)26Sortm9(CAGtdTomato)Hze/J mice, and Gt (ROSA)26Sortm4(ACTBtdTomato-EGFP)Luo/J mice were purchased from Jackson laboratories. For lineage tracing experiments, Lgr5-eGFPIRES-CreERT2 mice were crossed with B6.Cg-Gt (ROSA)26Sortm9(CAG-tdTomato)Hze/J mice to generate mice Lgr5eGFP-IRES-CreERT2; Rosa26-CAG-tdTomato heterozygote. The animals were maintained ad libitum and all studies were performed under the guidelines and protocols of the Institutional Animal Care and Use Committee of the University of Kansas Medical Center. All the animal experimental protocols were approved by the Institutional Animal Care and Use Committee of the University of Kansas Medical Center (ACUP number 2019-2487).

\section{Histology}

The rectum of each animal was dissected and washed in PBS to remove intestinal contents, and the rectum was fixed in $10 \%$ neutral-buffered formalin before paraffin embedding. Tissue was routinely processed and cut into $5-\mu \mathrm{m}$ sections for hematoxylin and eosin and immunohistochemical staining. All hematoxylin and eosin (HE) (Fisher Scientific, Pittsburgh, PA) staining was performed at the Pathology Core Facility in the KUMC Cancer Center.

To visualize rectal epithelial cell proliferation, each mouse was injected intraperitoneally with $120 \mathrm{mg} \mathrm{kg}^{-1}$ BrdU (Sigma-Aldrich, USA) 2 to $4 \mathrm{~h}$ before killing, and rectum was collected for paraffin embedding and BrdU immunohistochemistry. Tissue sections were routinely deparaffinized and rehydrated through graded alcohols and incubated overnight at room temperature with a biotinylated monoclonal BrdU antibody (Zymed, South Francisco, CA). Nuclear staining was visualized using streptavidin-peroxidase and diaminobenzidine (DAB), and samples were lightly counterstained with hematoxylin. Rectum from mice, not injected with BrdU, was used as a negative control. Digital photographs of rectal crypts were taken at high $(\times 40-60)$ magnification (Zeiss AxioHOME microscope), and crypt epithelial cells in intestinal sections 
were examined using Image software and classified as BrdU positive if they grossly demonstrated brown-stained nuclei from DAB staining or as BrdU negative if they were blue-stained nuclei.

\section{Determination of crypt depth}

Crypt depth was independently and objectively analyzed and quantitated in a blind manner from coded digital photographs of crypts from HE-stained slides using ImageJ 1.37 software to measure the height in pixels from the bottom of the crypt to the top. This measurement in pixels was converted to length (in $\mu \mathrm{m}$ ) by dividing with the following a conversion factor $\left(1.46\right.$ pixels $\left.\mu \mathrm{m}^{-1}\right)$.

\section{Organoids culture}

Rectum from C57BL/6 and Gt (ROSA)26Sortm4(ACTBtdTomato-EGFP)Luo/J was used for Crypt isolation and development of ex vivo organoid culture. Tissue was washed gently in cold PBS (calcium and magnesium free) to clean fat and stool. Tissue was cut approximately into $3-\mathrm{mm}$ pieces and washed with $10 \mathrm{ml}$ of cold PBS using a serological pipette. The pieces were allowed to settle by gravity and supernatant was discarded. This washing step was repeated few times until clear supernatant was visible. Clean rectal tissue was then incubated in Gentle Cell Dissociation Reagent (Stemcell Technologies \#07174) for $15 \mathrm{~min}$ at room temperature on a rocking platform (20 RPM). Once settled at the bottom of the tube, tissue pieces were resuspended in sterile $0.1 \%$ BSA containing PBS solution and pipetted up and down three times to release villi portion (fraction one). This step was repeated four times to release rectal crypts from the tissue pieces. These four fractions were passed through 70- $\mu \mathrm{m}$ filters (BD Biosciences) and centrifuged at $275 \mathrm{~g}$ for $5 \mathrm{~min}$ at $4{ }^{\circ} \mathrm{C}$, and single cells were discarded. Crypt pellet was resuspended in $10 \mathrm{ml}$ cold $\left(2-8{ }^{\circ} \mathrm{C}\right)$ DMEM-F12 and centrifugated at $200 \times g$ for $5 \mathrm{~min}$. Isolated crypts were resuspended in cold Matrigel (Corning \#356231) and cold media (StemCell Technologies IntestiCult $\left.^{\mathrm{mi}} \# 06005\right)$ in a 50:50 ratio. This was seeded as a dome in center of the wells of a 48-well plate previously warmed a $37^{\circ} \mathrm{C}$ to solidify the Matrigel. After $15 \mathrm{~min}$ of incubation at $37^{\circ} \mathrm{C}$ when Matrigel was solid, culture media was added from the side of the well. Plates were maintained at $37^{\circ} \mathrm{C}$ with $5 \% \mathrm{CO}_{2}$ with media change every 3 days.

\section{Irradiation procedure}

Pelvic irradiation in single or multiple fractions was performed on anesthetized mice (intraperitoneal injection of ketamine $(87.5 \mathrm{mg} / \mathrm{kg})$ and xylazine $(12.5 \mathrm{mg} / \mathrm{kg})$ cocktail) using XenX (Xstrahl, Life Sciences) $[6,16]$. A $3-\mathrm{cm}$ area of the mice containing pelvic region was irradiated (Fig. 1a), thus shielding the upper and middle abdomen, thorax, head, and neck as well as lower and upper extremities, protecting a significant portion of the bone marrow. The total irradiation time to deliver the intended dose was calculated with respect to dose rate, radiation field size, and fractional depth dose to ensure accurate radiation dosimetry.

\section{Organoid transplantation}

Rectal organoids from Lgr5-eGFP-IRES-CreERT2; Rosa26CAG-tdTomato mice were transplanted to C57Bl6 mice exposed to pelvic irradiation (24 Gy single fraction). Around 150-200 organoids were transplanted in mice rectum through anus using a micropipette. Following transplantation, recipient mice were treated with tamoxifen to activate Cre recombinase in transplanted cells.

\section{In vivo lineage tracing assay}

Lgr5-eGFP-IRES-CreERT2 mice were crossed with B6.Cg-Gt (ROSA)26Sortm9(CAG-tdTomato)Hze/J mice (Jackson Laboratories) [17] to generate Lgr5-eGFP-IRESCreERT2; Rosa26-CAG-tdTomato heterozygote. Involvement Lgr5 RSCs in rectal epithelial regeneration was examined by lineage tracing assay. Lineage tracing was induced by tamoxifen administration in Cre reporter mice to mark the RSCs and their respective tdT-positive progeny. Adult mice were injected with tamoxifen (Sigma) ( $9 \mathrm{mg}$ per $40 \mathrm{~g}$ of body weight, i.p.) to label Lgr5+ lineages.

\section{Immunocytochemistry}

To validate the organoids derived from rectum expression of rectal epithelial specific markers such as Muc2, ChgA, and CFTR were examined using immune-fluorescence analysis (supplement figure 1). In brief, rectal organoids were permeabilized with $0.1 \%$ Triton X-100 (Sigma \#9002-93-1) in PBS for $30 \mathrm{~min}$ at room temperature, washed twice with PBS, and blocked with 5\% normal goat serum (Life Technologies \#50062Z) for $60 \mathrm{~min}$. Organoids were incubated with primary antibodies (1:50 anti CFTR (Mouse IgM, Abcam \#ab2784), 1:100 anti MUC2 (Rabbit IgG, Novus Biological \#NBP1-31231) overnight at $4{ }^{\circ} \mathrm{C}$ followed by incubation with secondary antibodies (antiIgG Rabbit Alexa Fluor 488 (Invitrogen \#A11008) $8 \mu \mathrm{g} /$ ml, 1:50 anti-IgM mouse Dylight 594 (Abcam \#ab97009)) for $5 \mathrm{~h}$ at room temperature. For phospho-Histone H2A.X detection, organoids were stained with the Anti-phosphoHistone H2A.X (Ser139) antibody $(2 \mu \mathrm{g} / \mathrm{ml})$ (Mouse IgG1, Millipore \#16-202A, clone JBW301, FITC conjugate).

\section{ATP uptake-cell viability assay}

This assay is designed for determining cell viability in 3D microtissue/organoid [18]. Cell viability of the 3D organoid was measured using the CellTiter-Glo ${ }^{\circ} 3 \mathrm{D}$ kit (Cat no.\#. G968A, Promega, Madison-WI) using the manufacturer's instruction. In this assay, the reagent 

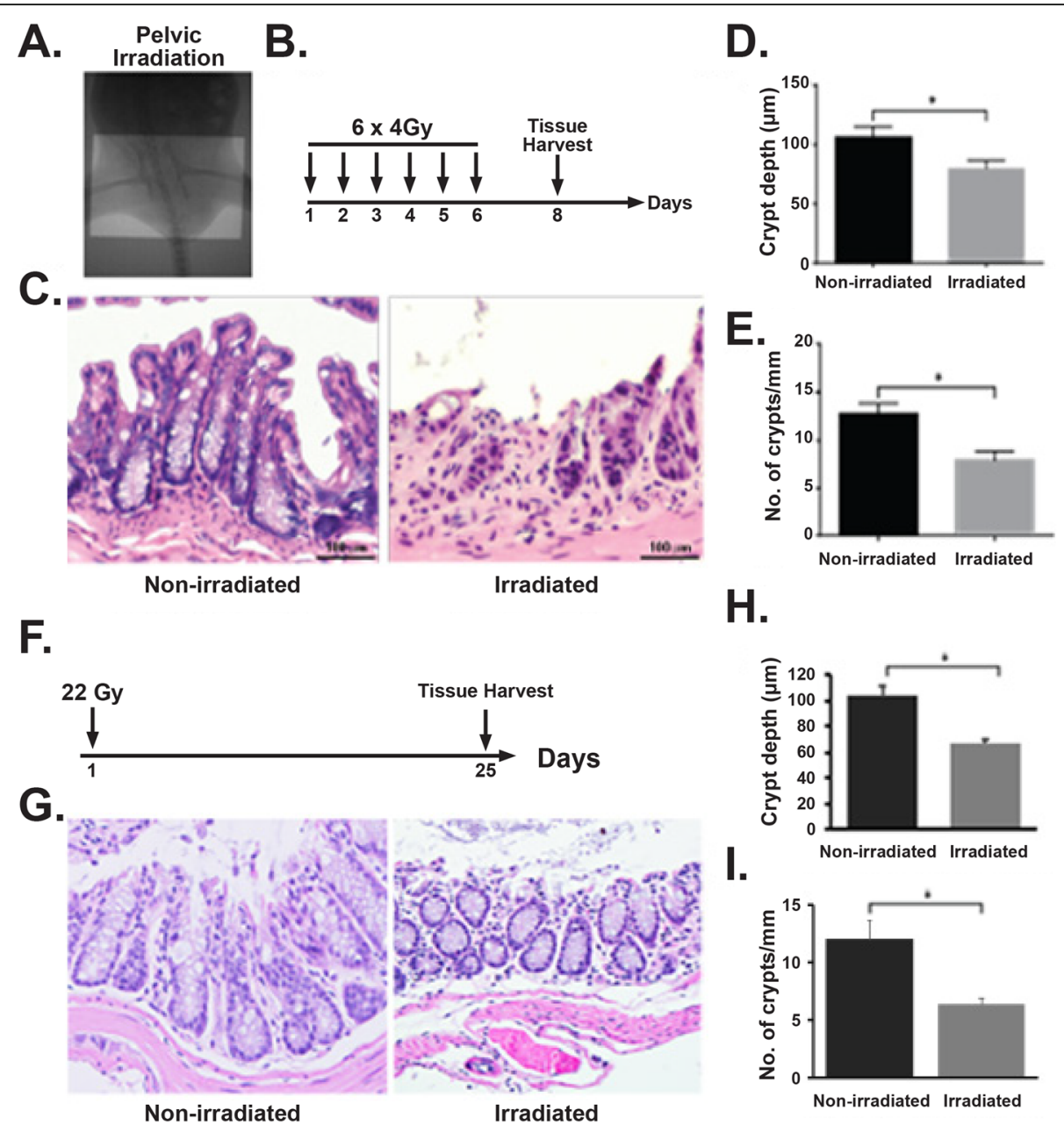

H.
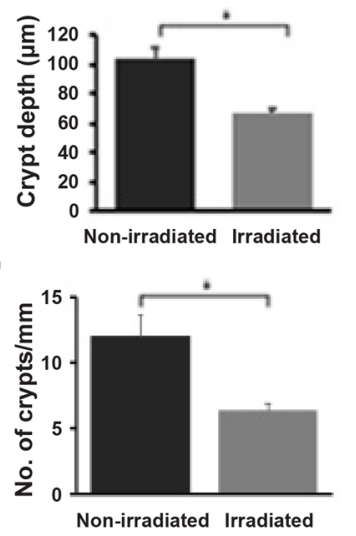

Fig. 1 Radiation-induced damage to rectal tissue. a Portal camera image demonstrating pelvic irradiation (PIR) exposure field. b Schematic diagram demonstrating the timeline of fractionated pelvic irradiation. $\mathbf{c}$ H\&E-stained representative cross section of the rectum. Non-irradiated mice showed a normal structure of crypts. However, mice exposed to fractionated pelvic irradiation suffer from crypt loss and epithelial damage. d, e Histogram demonstrating crypt depth (c) and number of crypt per $\mathrm{mm}(\mathbf{d})$ on rectal tissue. Exposure to fractionated pelvic irradiation significantly reduces crypt depth (d) ( $n 3, p<0.0061$, Mann-Whitney test) and number of crypt per mm ( $n$ 3, $p$ value 0.0061, Mann-Whitney test) on rectal tissue (e). $\mathbf{f}$ Schematic diagram demonstrating the timeline of single fraction pelvic irradiation. $\mathbf{g}$ H\&E-stained representative cross section of rectum. Non-irradiated mice showed a normal structure of crypts. However, mice exposed to pelvic radiation-induced epithelial damage with loss of crypt. h, i Histogram demonstrating mice exposed to pelvic irradiation decreases crypt depth ( $n$ 3, $p<0.0048$, Mann-Whitney test) and crypt/mm (n 3, p<0.0056, Mann-Whitney test) on rectal tissue

penetrates spheroids and using the lytic capacity accurately determines the viability by measuring the ATP uptake by the organoid. This $3 \mathrm{D}$ assay reagent measures ATP as an indicator of viability and generates a luminescent readout. In brief, organoids were grown for 3-4 days in opaque-walled 96-well plates suitable for 3D cell culture. Forty eight hours after irradiation, an equivalent volume of CellTiter-Glo ${ }^{\circ}$ 3D reagent was added in each well. The content of the plate was shaken for $5 \mathrm{~min}$ and luminescence was recorded $25 \mathrm{~min}$ after reagent addition. Different concentrations of ATP in water were plated at $100 \mu \mathrm{l}$ medium to generate an ATP standard curve. Luminescence of samples was compared to luminescence of standards to determine ATP detected by the CellTiter-Glo $3 \mathrm{D}$ Reagent in samples.

\section{RNAseq and gene expression analyses}

Flow-sorted Lgr5-positive and Lgr5-negative rectal epithelial cells were subjected to RNA sequencing. RNA samples were sent to BGI genomic center (China), and paired-end cDNA libraries were prepared and sequenced using a BGISEQ-500RS. Raw reads were filtered and trimmed according to their quality score profiles as implemented in PrinSeq v0.20.4 (trimming at minimum quality of 20; low complexity reads (dust) $=32$; no undetermined bases) [19]. High-quality reads were mapped 
against the mouse reference genome from NCBI (GCF 000001635.26_GRCm38.p6) using HISAT2 2.1.0 under default settings [20]. Mapped reads were converted to BAM format and sorted as in samtools [21]. We then assembled transcripts for each sample, estimated transcript abundances, and created table counts using StringTie 1.3.4d [22]. Transcript table counts were imported into the $\mathrm{R}$ environment using the tximport package. We performed a differential gene expression analysis using the negative binomial method with shrunk coefficients as implemented in the DESeq2 and package [23]. To explore enriched metabolic pathways, we performed a gene set analysis as implemented in the gage package [24], to then visualize such metabolic pathways in using the pathview package [25].

\section{Statistical analysis}

All images were analyzed using ImageJ software, and graphs were generated with GraphPad software. For histopathological and confocal images, rectum regions were chosen at random for digital acquisition for quantitation. Number of animals used for all in vivo and ex vivo experiments were $n=3$ per group. A Mann-Whitney test was used to determine significant differences between experimental conditions $(p<0.05)$ with representative standard errors of the mean.

\section{Result}

Rectal epithelium is sensitive to clinically relevant fractionated radiation

Rectal epithelium structurally differs from small intestine and colon. Histopathological analysis demonstrated that rectum has elongated crypt with very minimal/short villus like structure as they do not have absorptive function. In the present study, we have evaluated the effect of external beam fractionated radiation on mucosal epithelial structure of rectum. C57Bl6 mice were exposed to 6 fractions of 4 Gy pelvic irradiation (Fig. 1a, b) where each fraction was delivered in consecutive 6 days. At the second day after last fraction of radiation, rectum was subjected to histopathological analysis. H\&E staining demonstrated significant loss of crypt-like structure with reduction in crypt depth (Fig. 1c-e). To develop an acute rectal injury model mice has been exposed to single fraction of pelvic irradiation (22 Gy) (Fig. 1f). Histopathological analysis demonstrated significant damage in epithelium with the loss of crypt and reduction in crypt depth (Fig. 1g-i). These results clearly suggest that both fractionated radiotherapy nearly for a week as well as single fraction could promote acute injury in rectal epithelium loss of mucosal epithelial structure which may lead to severe pain/discomfort and blood loss.

\section{Radiation exposure reduces the regenerative capacity of} Lgr5+ve RSCs

Mucosal epithelial loss is primarily consisting of stem cell loss along with dysregulated stromal signal [5]. In this study, we have examined the effect of irradiation in rectal stem cell. To determine the effect of radiation in regenerative capacity of Lgr5+ve cells in rectum, Lgr5eGFP-IRES-CreERT2; Rosa26-CAG-tdTomato mice were exposed to high dose of single fraction of pelvic irradiation and then treated with single dose of tamoxifen (Fig. 2a). In irradiated mice, the number of tdT-positive cells which represents cells derived from Lgr5+ve RSCs significantly decreased compared to untreated control (Fig. 2b, c).

Next we have examined the effect of fractionated radiation in regenerative capacity of rectal epithelial cells. Brdu analysis demonstrated the presence of the reduced number of Brdu+ve proliferating cells in rectal crypt from mice exposed to fractionated irradiation compared to un-irradiated control (Fig. 3a-c). However, TUNEL staining demonstrated significant increase in epithelial apoptosis in response to fractionated radiation (Fig. 3d, e). Lgr5+ve RSCs are located at crypt bottom (Fig. 2b). It was observed that majority of TUNEL-positive cells were located in crypt bottom suggesting radiation-induced apoptosis in RSCs. To determine the regenerative capacity of Lgr5+ve RSCs in response to fractionated irradiation, Lgr5-eGFP-IRES-CreERT2; Rosa26-CAG-tdTomato mice were exposed to similar fractionated pelvic radiation dose regimen followed by tamoxifen injection (Fig. 3f). Confocal microscopic imaging demonstrated that significantly less number of tdTomato-positive cells in irradiated group compared to un-irradiated control (Fig. 3g, h).

\section{Fractionated irradiation inhibits rectal organoid growth}

Intestinal organoids primarily from small intestinal and colonic crypts have been used extensively to study radiation effect $[4,6,9,16,26,27]$. Organoids has also been considered as a model system to determine radiosensitivity for intestinal stem cells [27-29]. In this study for the first time, we have used organoids from mice rectum to study radiation toxicity. Organoids in response to irradiation demonstrated a significant decrease in survival in a radiation dose-dependent manner (Fig. 4a-b). We have also determined the effect of irradiation on overall survival of these organoids by using ATP assay. Irradiation reduces viability of these organoids in a dose-dependent manner (Fig. 4c). To determine the effect of clinically relevant fractionated doses of radiation, organoids were exposed to graded doses (2-8 Gy) of fractionated irradiation according to schedule described in Fig. 5a. Representative brightfield images demonstrated lethality in organoids when exposed to fractionated irradiation $(2 \mathrm{~Gy} \times 4)$ (Fig. $5 \mathrm{~b}$ ). Organoids demonstrated complete loss in budding structure and eventually manifested as cloud of cell debris at 
A.

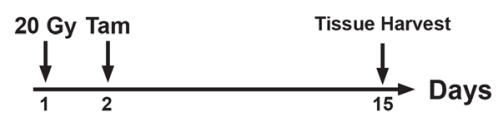

B.

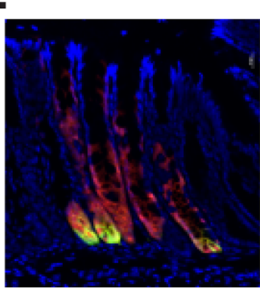

Non-irradiated
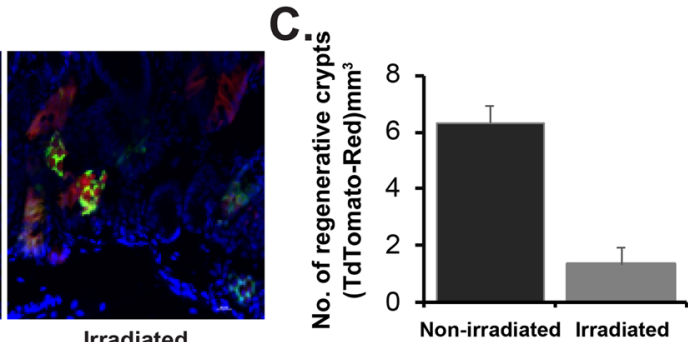

Fig. 2 Pelvic irradiation reduces the regenerative response of LGR5+ stem cells in rectal crypts. a Schematic representation of the treatment schema for lineage tracing assay in Lgr5-eGFP-IRES-CreERT2; Rosa26-CAG-tdTomato mice. b Confocal microscopic images of the rectum section from Lgr5-eGFP-IRES-CreERT2; Rosa26-CAG-tdTomato mice. tdTomato (tdT)-positive cells are shown in red; Lgr5+ GFP+ cells are shown in green. Nuclei are stained with DAPI (blue). Marked expansion of tdT-positive red cells representing transit amplifying cells in crypt (regenerative crypt) were noted with un-irradiated rectal tissue compared to mice exposed to pelvic irradiation. $\mathbf{c}$ The number of regenerative crypt significantly decreased in irradiated mice rectum compared to un-irradiated control $(n=3 ; p<0.007)$

day 9-11 post irradiation considering the days from first fraction of radiation (Fig. 5b). Analysis of Feret diameter (Fig. 5c), total organoid count (Fig. 5d), percent budding organoids (Fig. 5e), and viability percentage (Fig. 5f) clearly demonstrated a dose response with graded doses of fractionated irradiation. Feret diameter is used to determine the organoid size [30]. Organoids exposed to irradiation clearly shows reduction in organoid size. Total organoid count also reduced significantly with irradiation suggesting increase in death of organoids in a dose-dependent manner. Quantification of budding organoids showed a significant decrease in percentage of budding organoids in respect of total organoid count suggesting impaired growth and proliferation. These observations therefore suggest that rectal organoids which primarily depend on rectal stem cell for their survival are sensitive to fractionated irradiation. Moreover consistent dose-dependent response of rectal organoids against radiation suggests that ex vivo organoid platform could be used for radiation biodosimetry.

\section{Rectal stem cells in organoid is sensitive to irradiation}

To determine the radiosensitivity of RSCs, we have developed organoids from Lgr5-eGFP-IRES-CreERT2 mice and exposed to irradiation. Confocal microscopic imaging of un-irradiated organoids demonstrated the presence of Lgr5+ve cells at the tip of the budding crypt-like structure. However, in response to single (Fig. $4 \mathrm{~d}$, e) or multiple fraction of irradiation (Fig. $5 \mathrm{~g}, \mathrm{~h}$ ) $(2$ Gy $\times 4$ ), organoids demonstrated loss of Lgr5+ve cells with significant damage in overall organoid structure. Within days after last fraction of irradiation, organoids demonstrated complete lethality as organoid structure end up as cloud of cell debris. These time course studies clearly suggest that radiation-induced loss of Lgr5+ve RSCs is the earlier event which results complete inhibition of organoid growth and proliferation and eventual lethality.

\section{Radiation induces DNA damage in rectal organoids}

In response to DNA double-strand break (DSB), H2AX, the histone $\mathrm{H} 2 \mathrm{~A}$ variant, becomes phosphorylated at serine 139. The phosphorylated form of $\mathrm{H} 2 \mathrm{AX}$ is also known as $\gamma \mathrm{H} 2 \mathrm{AX}$. To determine DNA damage response in irradiated rectal organoids, the number of $\gamma \mathrm{H} 2 \mathrm{AX}$ foci was quantified. Organoids stained with anti- $\gamma \mathrm{H} 2 \mathrm{AX}$ antibody demonstrated a significant increase in the number of $\gamma \mathrm{H} 2 \mathrm{AX}$ foci following exposure to fractionated irradiation in a dose-dependent manner (Fig. 6a, b). To further validate the involvement of DNA damage in loss of organoid growth and survival organoids were treated with doxorubicin, a DNA damaging agent. A significant increase in $\gamma \mathrm{H} 2 \mathrm{AX}$ foci was observed in doxorubicin-treated organoids compared to untreated control (Fig. 6c, d). Similar to irradiation, doxorubicin treatment also significantly reduces organoid growth and survival (Fig. 6e-g).

\section{Transplantation of rectal organoids mitigate radiation injury in rectum}

Both single or multiple fraction radiation demonstrated significant loss of RSCs with impaired regeneration of rectal epithelium. Therefore, restitution of Lgr5 RSCs is critical for repair and regeneration. Transplanting organoid cells as a regenerative therapy has been used against degenerative diseases in intestine [31-33]. However, organoid-based transplantation to mitigate radiation injury has not been examined in pre-clinical and clinical 
A.

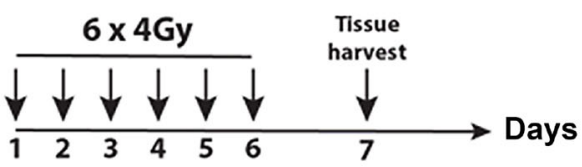

B.



D.
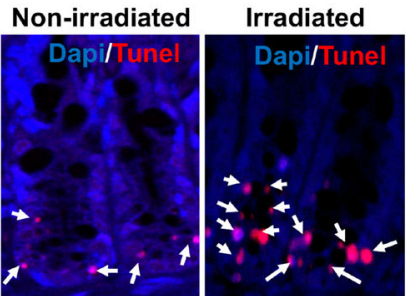

C.

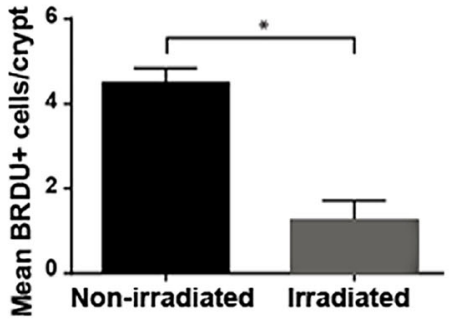

E.

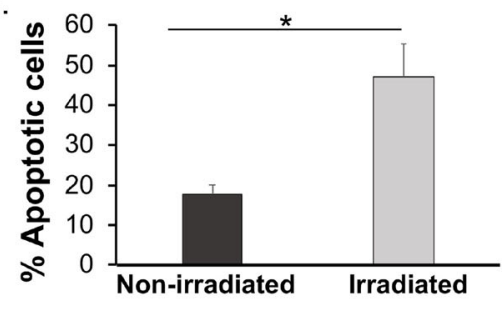

F.



G.



$\mathrm{H}$.

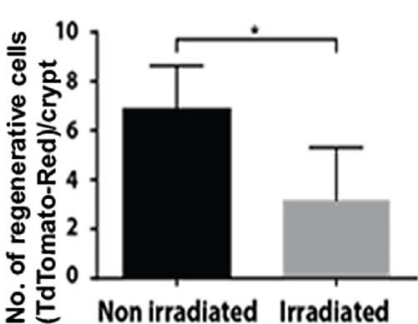

Fig. 3 Fractionated pelvic irradiation impairs rectal epithelial regeneration. a Schematic representation of the fractionated pelvic irradiation timeline. b Representative Brdu immunohistochemistry of mice rectum section. Note the decrease in Brdu-positive cells (stained brown, indicated with arrow) in irradiated cohort. c Histogram showing mean Brdu-positive cells per crypt. Fractionated pelvic irradiation significantly reduces Brdu-positive cells compared to un-irradiated control ( $p<0.0007$, Mann-Whitney test). $\mathbf{d}$ Representative TUNEL staining of mice rectum section. Note the increase in TUNEL-positive cells (stained red, indicated with arrow) in irradiated cohort. e Histogram showing mean TUNEL-positive cells per crypt. Fractionated pelvic irradiation significantly increased TUNEL-positive cells compared to un-irradiated control $(p<0.004$, Mann-Whitney test). $\mathbf{f}$ Schematic representation of the fractionated pelvic irradiation and tamoxifen treatment timeline. $\mathbf{g}$ Confocal microscopic images of the rectum section from Lgr5-eGFP-IRES-CreERT2; Rosa26-CAG-tdTomato mice. tdTomato (tdT)-positive cells are shown in red; Lgr5+ GFP+ cells are shown in green. Nuclei are stained with DAPI (blue). Marked expansion of tdT-positive red cells representing transit amplifying cells (regenerative cells) in crypt were noted with un-irradiated rectal tissue compared to mice exposed to pelvic irradiation. $\mathbf{h}$ The number of regenerative cells reduced significantly in irradiated mice rectum compared to un-irradiated mice $(p<0.0381$, Mann-Whitney test)

level. In the current manuscript, ex vivo rectal organoids grown from Lgr5-eGFP-IRES-CreERT2; Rosa26-CAGtdTomato mice were transplanted to C57Bl6 mice exposed to pelvic irradiation (24 Gy single fraction) (Fig. 7a). Presence of Lgr5+GFP+tdT+ was observed in C57Bl6 mice rectum receiving organoid transplant (Fig. $7 \mathrm{~b}, \mathrm{c}$ ). Transplantation of organoids from Lgr5-eGFP-IRESCreERT2; Rosa26-CAG-tdTomato having inducible Cre recombinase demonstrated presence of Lgr5+ cell-derived tdT-positive daughter cells in recipient C57Bl6 mice after tamoxifen treatment (Fig. 7b, c) suggesting regenerative response of RSCs. Histological analysis demonstrated loss of crypt and normal epithelial structure in irradiated rectum (Fig. 7d). However, mice receiving organoid transplant post irradiation demonstrated presence of crypt with normal epithelial structure (Fig. 7d). These results clearly 




B.

D.

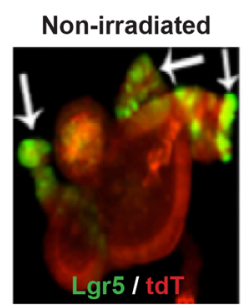

Irradiated

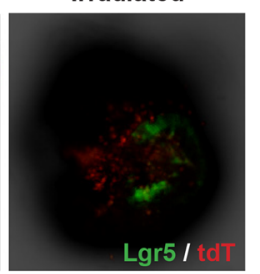

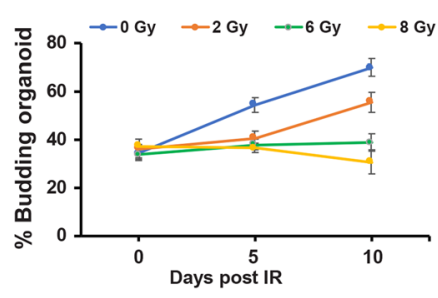

C.

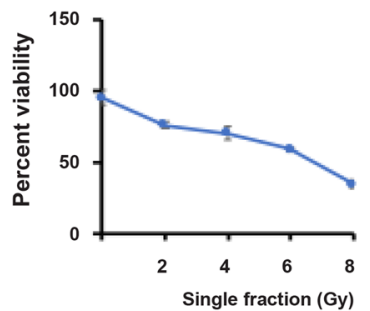

E.

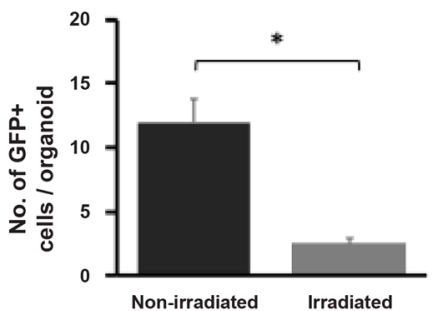

Fig. 4 Effect of irradiation on rectal organoids. a, b Microscopic image (phase contrast) of rectal organoids along with histogram of \% budding organoid demonstrating that irradiation impaired the organoid growth compared to un-irradiated control (2 Gy * $p<0.004,6 \mathrm{~Gy}{ }^{*} p<0.006$ ). Microscopic image with $\times 10$ (indicated with arrow) and $\times 40$ magnification demonstrated loss of budding crypt in irradiated organoids. $\mathbf{c}$ Survival assay (ATP uptake assay) demonstrated significant reduction of organoid survival in a dose-dependent manner. d Confocal microscopic images of organoids developed from Lgr5-EGFP-CRE-ERT2; R26- ACTB-tdTomato-EGFP mice demonstrated loss of Lgr5+ve cells (Green) in budding crypt from irradiated organoids compared to un-irradiated control. tdTomato is constitutively expressed in these mice as membranebound protein therefore allows better visualization of cellular morphology. e Histogram demonstrating significant decrease in Lgr $5+$ ve cells in irradiated rectal organoids compared to un-irradiated control $(p<0.0004)$

suggest that organoid transplantation can induce repair and regeneration of irradiated rectal epithelium with the engraftment of regenerative RSCs. As expected, no mortality was observed following PIR; however, mice without organoid transplant showed loss in body weight (Fig. 7e). Mice receiving organoid transplant post PIR improved their body weight compared to untreated mice (Fig. 7e). Moreover, immunofluorescence analysis of rectal tissue demonstrated increase in Ki67+ proliferating cells (Fig. 7f, g) and a significant decrease in apoptotic cells (Fig. 7h, i) in this group suggesting that organoid transplantation increases regenerative response in epithelium with inhibition of PIR-induced apoptosis.

\section{LGR5-positive cells expresses distinct stem cell marker in rectal crypts}

Although ISCs were well characterized in small intestine and colon their rectal counterpart has not been studied well. LGR5 is a classic stem cell marker for intestinal stem cells localized in the bottom of crypts in small bowel and colon. These cells also express several other stem cell markers such as prominin1, musashi, Ascl2, and OLMF4 [34]. In the current report, using Lgr5-Cre-ERTGFP mice, we have already shown that Lgr5+ve cells were present in crypt bottom of rectum and responsible for regenerative response of rectal epithelium. To determine the presence of other known stem cell marker, we have performed RNAseq analysis of sorted Lgr5+ve cells and Lgr5-ve cells (Fig. 8a). We were able to identify 801 genes differentially expressed between these two populations (Fig. 8b). Among these genes, Lgr5-positive cells also expresses a series of known stem cell markers previously identified in intestines and other tissues. However, we have identified 9 markers which exclusively expressed in rectal Lgr5-positive cells compared to same cell types in small and large intestine (Fig. 8c). Pathway-based analysis suggested presence of cell cycle, DNA repair, and recombination-related genes in Lgr5+ve cells (Fig. 8d).

\section{Discussion}

Results of the current study both in mice model and ex vivo organoid model indicate that Lgr5+ve rectal 


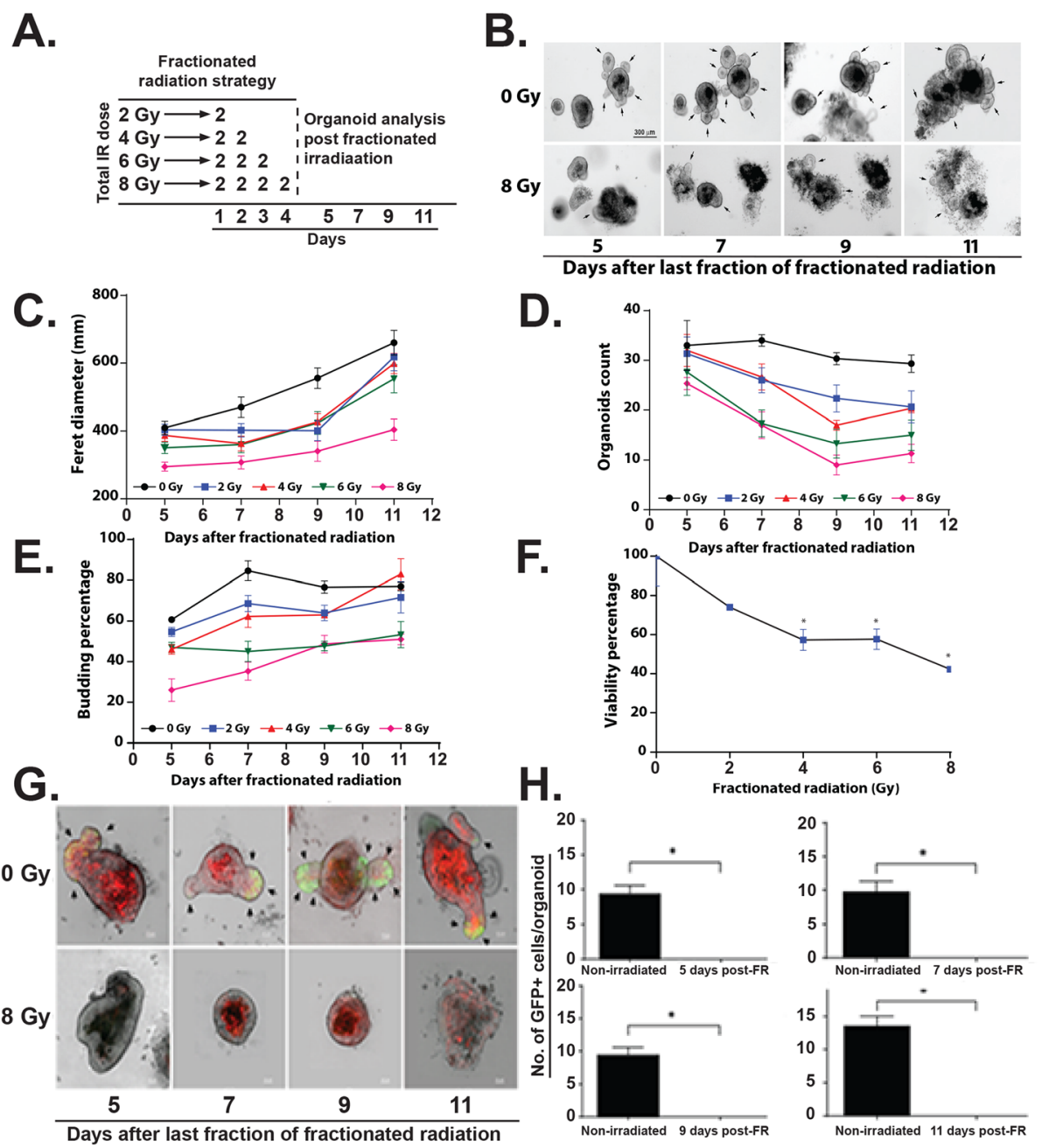

Fig. 5 Effect of fractionated radiation on rectal organoids. a Schematic representation of the fractionated irradiation to organoid. $\mathbf{b}$ Microscopic image (phase contrast) of rectal organoids. c-e Significant decrease in physical parameters such as c Feret diameter ( 8 Gy vs 0 Gy $(p<0.0001)$ at day 8 post IR), $\mathbf{d}$ organoid count ( 8 Gy vs 0 Gy $(p<0.0028$ ) at day 8 post IR), and e budding percentage ( 8 Gy vs 0 Gy $(0.0094)$ at day 8 post IR) was observed in a dose-dependent manner. $\mathbf{f}$ Significant decrease in organoid viability was observed in a dose-dependent manner ( 8 Gy vs 0 Gy (0.0028) Mann-Whitney test). $\mathbf{g}$ Confocal microscopic images of organoids developed from Lgr5-EGFP-CRE-ERT2; R26-ACTB-tdTomato-EGFP mice demonstrated loss of Lgr5+ve cells (Green) in budding crypt from irradiated organoids compared to un-irradiated control. tdTomato is constitutively expressed in these mice as membrane-bound protein therefore allows better visualization of cellular morphology. $\mathbf{h}$ Histogram demonstrating significant decrease in Lgr5+ve cells in irradiated rectal organoids compared to un-irradiated control at different time point post irradiation $(p<0.0079$, day 11 post IR)

stem cell is sensitive to acute radiation injury. We have generated an in vitro radiation sensitivity assay in organoid model. Findings of this study provide strong evidence in support of our in vivo data that clinically relevant fractionated irradiation cause acute loss of rectal stem cell in rectal epithelium which leads to rectal epithelial damage. Organoid-based studies clearly demonstrated a dose-dependent effect which is consistent both in multi- and single fraction of irradiation. Current literatures primarily focused on the radiation-induced rectal fibrosis which is a late effect of radiation toxicity [7]. However, it is very critical to analyze the initial cause of onset of fibrosis. Our study demonstrates that clinical doses of pelvic irradiation impaired the rectal epithelial homeostasis and regeneration. Previous reports suggested loss of mucosal epithelium may promote repopulation of inflammatory cells and later fibrotic tissue [35].

Unlike small intestine and colon, characterization of rectal stem cell has not been studied extensively. Our previous reports have demonstrated that Lgr5+ve stem cells in small intestine are radiosensitive and disappear within $72 \mathrm{~h}$ after a lethal dose of irradiation [4-6]. We have also shown that therapeutic intervention will only be successful to mitigate radiation-induced acute 


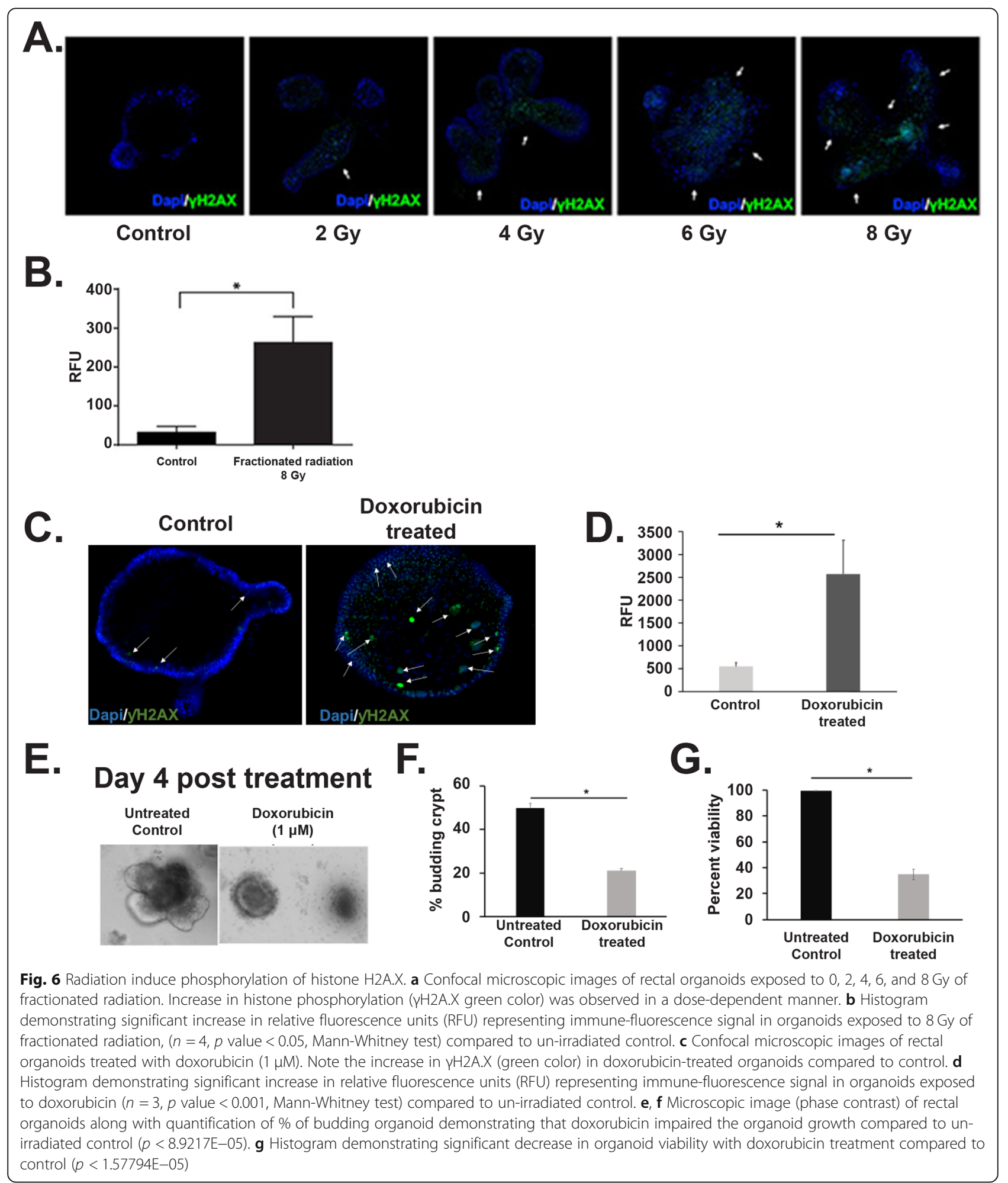

mucosal damage if applied within $72 \mathrm{~h}$ post irradiation when endogenous Lgr5+ve stem cells are present [5]. Failure of mitigation in later time points clearly suggests that presence of Lgr5+ve stem cells are critical for the rebuilding process. In another study from our group demonstrated that small molecule mediated activation Lgr5+ve stem cells in small intestine promote repair and regeneration [6]. In the present study, we have shown 


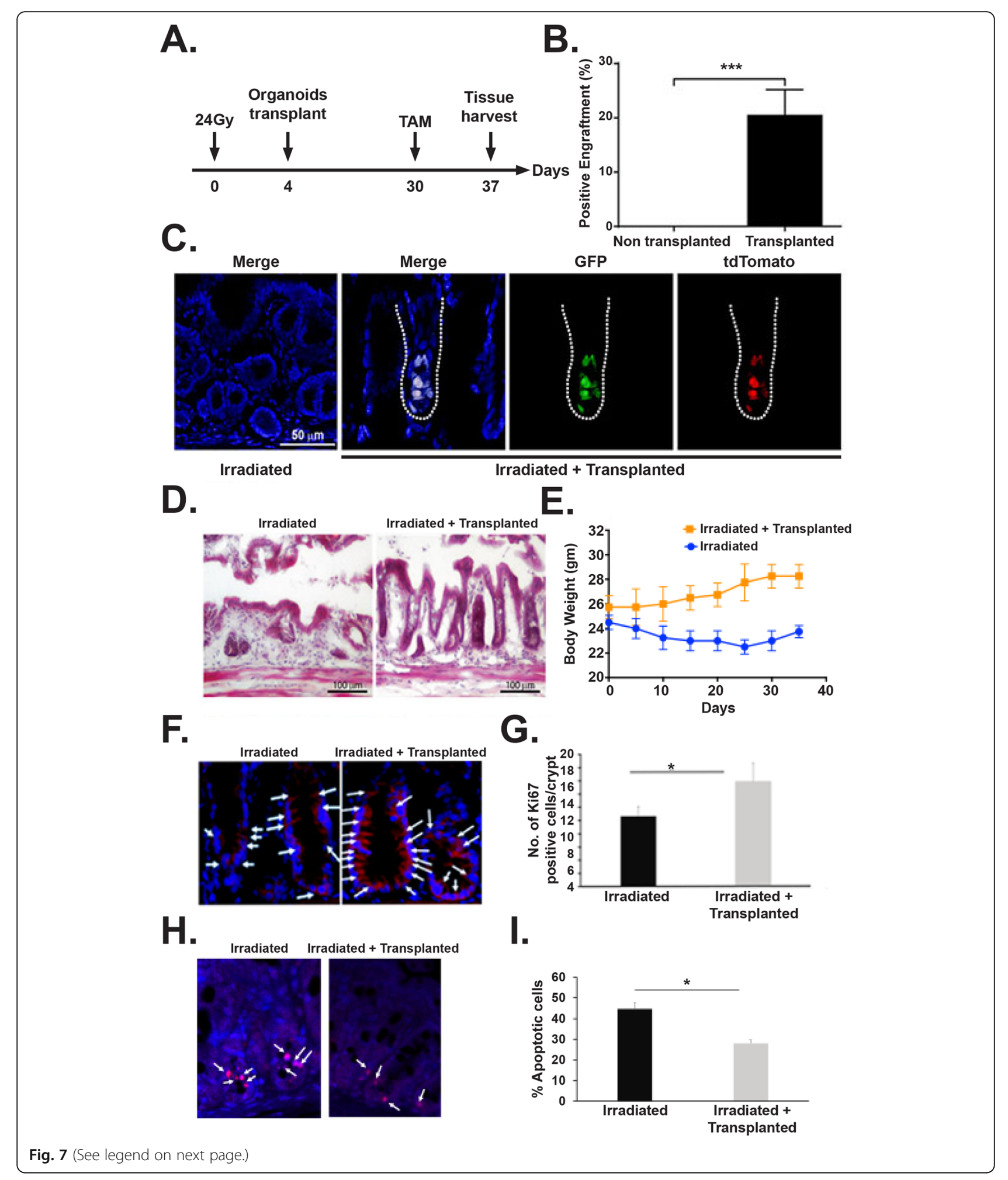


(See figure on previous page.)

Fig. 7 Transplantation of rectal organoids a Schematic diagram demonstrating pelvic irradiation and organoid transplantation time line. b Histogram demonstrating significant presence of tdT cell containing microscopic fields (positive fields) in transplanted mice rectal epithelium compared to non-transplanted control ( $p<0.0005$, Mann-Whitney test, $n=4$ mice (5-7 fields were counted per mice)). c Confocal microscopic image of rectal epithelium receiving transplanted organoids. Please note presence of GFP+ve (green) and tdTomato (red) cells in transplanted rectum suggesting presence of transplanted cells from Lgr5-eGFP-IRES-CreERT2; Rosa26-CAG-tdTomato mice rectal organoid. Merged figure demonstrated co-expression of Lgr5+GFP and reporter tdTomato. $\mathbf{d}$ HE staining demonstrated rectal epithelial repair in transplanted mice compared to non-transplanted control. e Measurement of body weight in mice receiving PIR with/without organoid transplant. Significant improvement in body weight was noted in mice receiving organoid transplant. $\mathbf{f}$ Representative Ki67 immunofluorescence staining of mice rectum section. Note the increase in Ki67-positive cells (stained red, indicated with arrow) in mice receiving organoid transplant. $\mathbf{g}$ Histogram showing mean Ki67-positive cells per crypt. Organoid transplantation significantly increases Ki67-positive cells compared to untreated control ( $p<0.002$, Mann-Whitney test). $\mathbf{h}$ Representative TUNEL staining of mice rectum section. Note the decrease in TUNEL-positive cells (stained red, indicated with arrow) in mice receiving organoid transplant. i Histogram showing mean TUNEL-positive cells per crypt. Organoid transplantation significantly decrease TUNEL-positive cells compared to untreated control ( $p<0.0001$, Mann-Whitney test)

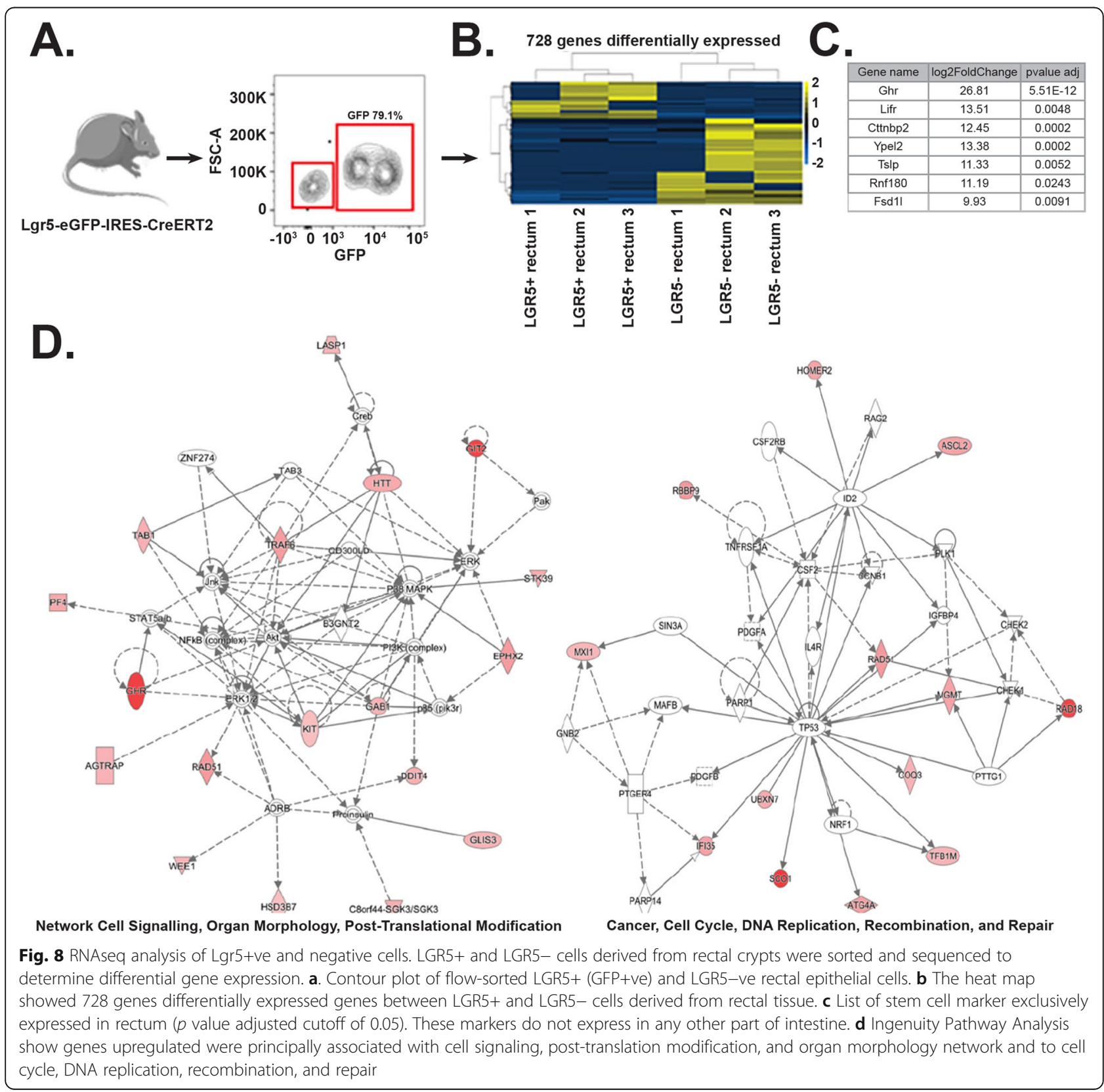


that Lgr5+ve cells functions as stem cells in rectum and participate in epithelial homeostasis and regeneration. Radiation-induced depletion of these stem cells inhibits the regenerative process. Lineage tracing assay in mice demonstrated that in response to fractionated irradiation regenerative potential of these Lgr5+ve cells compromised significantly.

Radiation toxicity primarily induced in the cell by DNA damage. Our study demonstrates that fractionated doses of irradiation induce the DNA damage in organoid in a dose-dependent manner. Delivery of irradiation in multiple fractions with interval keeps level of DNA damage which promotes lethality in stem cells resulting organoid death. These results provide a mechanistic evidence of rectal epithelial damage when exposed to clinically relevant fractionated pelvic radiotherapy. To mitigate radiation-induced rectal epithelial damage, transplantation of rectal organoids enriched in rectal stem cells demonstrated repair and regenerative effect with the inhibition of radiation-induced apoptosis. Transplantation of organoids from Lgr5-eGFP-IRESCreERT2; Rosa26-CAG-tdTomato having inducible Cre recombinase demonstrated presence of $\mathrm{tdT}+$ cells in host rectal epithelium suggesting proliferation of Lgr5+ ve cells. Histopathological evidences of rectal epithelial repair also suggested that organoid transplantation not only induce the repair process by engraftment/cell replacement but may also release paracrine signals to induce the regenerative response of host stem/progenitor cells in irradiated rectum.

Previous reports showed that Lgr5+ cells in small intestine and colon shares other stem cell markers [34]. Our RNAseq data demonstrated that Lgr5+ cells in rectum shares a different set of stem cell markers compared to small intestine and colon. This data suggests that Lgr5+ cells in rectum may be different compared to other part of intestine and needs further investigation for their molecular and functional characterization. Previous reports demonstrated the presence of reserve and active pool of stem cells in small and large intestine which also differs in radiosensitivity. It is possible that there could be a reserve stem cell pool in rectum such as Krt-19+ve cells [36] observed in small intestine or colon. Further studies are needed to determine the presence of other stem cell pool.

\section{Conclusion}

In conclusion, the present study has demonstrated that Lgr5+ve RSCs are radiosensitive and it is critical to rescue these cells for repair and regeneration of rectal epithelium. Therefore, to ameliorate the acute toxicity in rectum in cancer patients undergoing pelvic radiotherapy, a RSC targeted therapy is needed.

\section{Supplementary Information}

The online version contains supplementary material available at https://doi. org/10.1186/s13287-020-02111-w.

\section{Additional file 1.}

\section{Abbreviations}

ISC: Intestinal stem cells; RSC: Rectal stem cells; PBI: Partial body irradiation; HE: Hematoxylin and eosin

\section{Acknowledgements \\ The authors acknowledge the histopathology core facility and confocal microscopy core facility of KUMC for their help and support.}

\section{Authors' contributions}

F.T. conceived and designed the experiments, performed the experiments, analyzed the data, and wrote the paper. P.B. conceived and designed the experiments and performed the experiments. E.C.N analyzed the data. X.D.O performed the experiments. C.S performed the experiments. S.S. contributed reagents/materials/analysis tools, conceived and designed the experiments, and wrote the paper. All authors read and approved the final manuscript.

\section{Funding}

This work was supported by KUMC Department of Radiation Oncology start up fund (S.S.), KUCC support Fund (S.S.).

\section{Availability of data and materials}

The authors declare that all data supporting the findings of this study are available within the article and its Supplementary Information files or from the corresponding author on reasonable request.

\section{Ethics approval and consent to participate}

Use of human subject, data or tissue: NA.

All animal studies were performed under the guidelines and protocols of the Institutional Animal Care and Use Committee of the Kansas University Medical Center. All the animal experimental protocols were approved by Institutional Animal Care and Use Committee of the University of Kansas medical center (ACUP Number: 2019-2487).

\section{Consent for publication}

Not applicable.

\section{Competing interests}

The authors declare no non-financial as well as financial conflicts of interest exist for any of the authors.

\section{Author details}

${ }^{1}$ Department of Radiation Oncology, University of Kansas Medical Center 3901 Rainbow Boulevard, Kansas City, KS 66160, USA. ${ }^{2}$ Center for

Bioinformatics and Integrative Biology, Facultad de Ciencias de la Vida, Universidad Andres Bello, Santiago, Chile. ${ }^{3}$ Department of Cancer Biology, University of Kansas Medical Center, 3901 Rainbow Boulevard, Kansas City, KS 66160, USA.

Received: 21 July 2020 Accepted: 21 December 2020

Published online: 15 January 2021

\section{References}

1. Anscher MS, Chang MG, Moghanaki D, Rosu M, Mikkelsen RB, Holdford D, Skinner V, Grob BM, Sanyal A, Wang A, Mukhopadhyay ND. A phase II study to prevent radiation-induced rectal injury with lovastatin. Am J Clin Oncol. 2018;41(6):544-8.

2. Jemal A, Bray F, Center MM, Ferlay J, Ward E, Forman D. Global cancer statistics. CA Cancer J Clin. 2011;61(2):69-90.

3. Michalski JM, Gay H, Jackson A, Tucker SL, Deasy JO. Radiation dose-volume effects in radiation-induced rectal injury. Int J Radiat Oncol Biol Phys. 2010; 76(3 Suppl):S123-9.

4. Saha S, Aranda E, Hayakawa Y, Bhanja P, Atay S, Brodin NP, Li J, Asfaha S, Liu L, Tailor Y, Zhang J, Godwin AK, Tome WA, Wang TC, Guha C, Pollard JW. Macrophage-derived extracellular vesicle-packaged WNTs 
rescue intestinal stem cells and enhance survival after radiation injury. Nat Commun. 2016;7:13096.

5. Saha S, Bhanja P, Kabarriti R, Liu L, Alfieri AA, Guha C. Bone marrow stromal cell transplantation mitigates radiation-induced gastrointestinal syndrome in mice. PLoS One. 2011;6(9):e24072.

6. Bhanja P, Norris A, Gupta-Saraf P, Hoover A, Saha S. BCN057 induces intestinal stem cell repair and mitigates radiation-induced intestinal injury. Stem Cell Res Ther. 2018;9(1):26.

7. Tabaja L, Sidani SM. Management of radiation proctitis. Dig Dis Sci. 2018; 63(9):2180-8.

8. Vanneste $B G$, Van De Voorde L, de Ridder RJ, Van Limbergen EJ, Lambin P, van Lin EN. Chronic radiation proctitis: tricks to prevent and treat. Int J Color Dis. 2015;30(10):1293-303.

9. Sato $T$, Vries RG, Snippert HJ, van de Wetering M, Barker N, Stange DE, van Es JH, Abo A, Kujala P, Peters PJ, Clevers H. Single Lgr5 stem cells build crypt-villus structures in vitro without a mesenchymal niche. Nature. 2009; 459(7244):262-5.

10. Yan KS, Chia LA, Li X, Ootani A, Su J, Lee JY, Su N, Luo Y, Heilshorn SC, Amieva MR, Sangiorgi E, Capecchi MR, Kuo CJ. The intestinal stem cell markers Bmi1 and Lgr5 identify two functionally distinct populations. Proc Natl Acad Sci U S A. 2012;109(2):466-71.

11. Takeda N, Jain R, LeBoeuf MR, Wang Q, Lu MM, Epstein JA. Interconversion between intestinal stem cell populations in distinct niches. Science. 2011; 334(6061):1420-4

12. van der Flier $L G$, Haegebarth $A$, Stange $D E$, van de Wetering $M$, Clevers $H$. OLFM4 is a robust marker for stem cells in human intestine and marks a subset of colorectal cancer cells. Gastroenterology. 2009;137(1):15-7.

13. Buczacki SJ, Zecchini HI, Nicholson AM, Russell R, Vermeulen L, Kemp R, Winton DJ. Intestinal label-retaining cells are secretory precursors expressing Lgr5. Nature. 2013;495(7439):65-9.

14. Wong W, Stange DE, Page ME, Buczacki S, Wabik A, Itami S, van de Wetering M, Poulsom R, Wright NA, Trotter MW, Watt FM, Winton DJ, Clevers $\mathrm{H}$, Jensen KB. Lrig1 controls intestinal stem-cell homeostasis by negative regulation of ErbB signalling. Nat Cell Biol. 2012;14(4):401-8.

15. Metcalfe C, Kljavin NM, Ybarra R, de Sauvage FJ. Lgr5+ stem cells are indispensable for radiation-induced intestinal regeneration. Cell Stem Cell. 2014;14(2):149-59.

16. Nag D, Bhanja P, Riha R, Sanchez-Guerrero G, Kimler BF, Tsue T, Lominska C, Saha S. Auranofin protects intestine against radiation injury by modulating p53/p21 pathway and radiosensitizes human colon tumor. Clin Cancer Res. 2019;25(15):4791-807.

17. Madisen L, Zwingman TA, Sunkin SM, Oh SW, Zariwala HA, Gu H, Ng LL, Palmiter RD, Hawrylycz MJ, Jones AR, Lein ES, Zeng H. A robust and highthroughput Cre reporting and characterization system for the whole mouse brain. Nat Neurosci. 2010;13(1):133-40.

18. Sendi H, Mead I, Wan M, Mehrab-Mohseni M, Koch K, Atala A, Bonkovsky $\mathrm{HL}$, Bishop CE. miR-122 inhibition in a human liver organoid model leads to liver inflammation, necrosis, steatofibrosis and dysregulated insulin signaling. Plos One. 2018;13(7):e0200847.

19. Schmieder R, Edwards R. Quality control and preprocessing of metagenomic datasets. Bioinformatics. 2011;27(6):863-4.

20. Kim D, Paggi JM, Park C, Bennett C, Salzberg SL. Graph-based genome alignment and genotyping with HISAT2 and HISAT-genotype. Nat Biotechnol. 2019;37(8):907-15.

21. Li H, Handsaker B, Wysoker A, Fennell T, Ruan J, Homer N, Marth G, Abecasis G, Durbin R, S. Genome Project Data Processing. The Sequence Alignment/ Map format and SAMtools. Bioinformatics. 2009;25(16):2078-9.

22. Pertea M, Kim D, Pertea GM, Leek JT, Salzberg SL. Transcript-level expression analysis of RNA-seq experiments with HISAT, StringTie and Ballgown. Nat Protoc. 2016;11(9):1650-67.

23. Love Ml, Huber W, Anders S. Moderated estimation of fold change and dispersion for RNA-seq data with DESeq2. Genome Biol. 2014;15(12):550.

24. Luo W, Friedman MS, Shedden K, Hankenson KD, Woolf PJ. GAGE: generally applicable gene set enrichment for pathway analysis. BMC Bioinformatics. 2009;10:161

25. Luo W, Brouwer C. Pathview: an R/Bioconductor package for pathway-based data integration and visualization. Bioinformatics. 2013;29(14):1830-1.

26. Wang Y, DiSalvo M, Gunasekara DB, Dutton J, Proctor A, Lebhar MS, Williamson IA, Speer J, Howard RL, Smiddy NM, Bultman SJ, Sims CE, Magness ST, Allbritton NL. Self-renewing monolayer of primary colonic or rectal epithelial cells. Cell Mol Gastroenterol Hepatol. 2017;4(1):165-182 e7.
27. Martin ML, Adileh M, Hsu KS, Hua G, Lee SG, Li C, Fuller JD, Rotolo JA, Bodo S, Klingler S, Haimovitz-Friedman A, Deasy JO, Fuks Z, Paty PB, Kolesnick RN. Organoids reveal that inherent radiosensitivity of small and large intestinal stem cells determines organ sensitivity. Cancer Res. 2020;80(5):1219-27.

28. Hua G, Wang C, Pan Y, Zeng Z, Lee SG, Martin ML, Haimovitz-Friedman A, Fuks Z, Paty PB, Kolesnick R. Distinct levels of radioresistance in Lgr5(+) colonic epithelial stem cells versus Lgr5(+) small intestinal stem cells. Cancer Res. 2017;77(8):2124-33.

29. Sato T, Stange DE, Ferrante M, Vries RG, Van Es JH, Van den Brink S, Van Houdt WJ, Pronk A, Van Gorp J, Siersema PD, Clevers H. Long-term expansion of epithelial organoids from human colon, adenoma, adenocarcinoma, and Barrett's epithelium. Gastroenterology. 2011;141(5): 1762-72.

30. Cruz-Acuna R, Quiros M, Farkas AE, Dedhia PH, Huang S, Siuda D, GarciaHernandez V, Miller AJ, Spence JR, Nusrat A, Garcia AJ. Synthetic hydrogels for human intestinal organoid generation and colonic wound repair. Nat Cell Biol. 2017;19(11):1326-35.

31. Cortez AR, Poling HM, Brown NE, Singh A, Mahe MM, Helmrath MA. Transplantation of human intestinal organoids into the mouse mesentery: a more physiologic and anatomic engraftment site. Surgery. 2018;164(4):643-50.

32. Jee J, Jeong SY, Kim HK, Choi SY, Jeong S, Lee J, Ko JS, Kim MS, Kwon MS, Yoo J. In vivo evaluation of scaffolds compatible for colonoid engraftments onto injured mouse colon epithelium. FASEB J. 2019;33(9):10116-25.

33. Okamoto R, Shimizu H, Suzuki K, Kawamoto A, Takahashi J, Kawai M, Nagata S, Hiraguri Y, Takeoka S, Sugihara HY, Yui S, Watanabe M. Organoid-based regenerative medicine for inflammatory bowel disease. Regen Ther. 2020;13:1-6.

34. Barker N, Bartfeld S, Clevers H. Tissue-resident adult stem cell populations of rapidly self-renewing organs. Cell Stem Cell. 2010;7(6):656-70.

35. Liang J, Zhang Y, Xie T, Liu N, Chen H, Geng Y, Kurkciyan A, Mena JM, Stripp BR, Jiang D, Noble PW. Hyaluronan and TLR4 promote surfactant-protein-Cpositive alveolar progenitor cell renewal and prevent severe pulmonary fibrosis in mice. Nat Med. 2016:22(11):1285-93.

36. Asfaha S, Hayakawa Y, Muley A, Stokes S, Graham TA, Ericksen RE, Westphalen CB, von Burstin J, Mastracci TL, Worthley DL, Guha C, Quante M, Rustgi AK, Wang TC. Krt19(+)/Lgr5(-) cells are radioresistant cancer-initiating stem cells in the colon and intestine. Cell Stem Cell. 2015;16(6):627-38.

\section{Publisher's Note}

Springer Nature remains neutral with regard to jurisdictional claims in published maps and institutional affiliations.

Ready to submit your research? Choose BMC and benefit from:

- fast, convenient online submission

- thorough peer review by experienced researchers in your field

- rapid publication on acceptance

- support for research data, including large and complex data types

- gold Open Access which fosters wider collaboration and increased citations

- maximum visibility for your research: over $100 \mathrm{M}$ website views per year

At $\mathrm{BMC}$, research is always in progress.

Learn more biomedcentral.com/submission 